\title{
Gender differences in predictors of self-rated health among older adults in Brazil and Chile
}

\author{
Ana Cristina Viana Campos ${ }^{1 *}$, Cecilia Albala², Lydia Lera² ${ }^{2}$ Hugo Sánchez ${ }^{2}$, Andréa Maria Duarte Vargas ${ }^{3}$ \\ and Efigênia Ferreira e Ferreira ${ }^{3}$
}

\begin{abstract}
Background: The determinants of self-rated health (SRH) have been widely investigated to explain social differences and gender differences in health. This study aimed to investigate the gender differences in predictors of SRH among Brazilian and Chilean older adults.

Methods: We used two samples of older people: 2052 Brazilian community-dwelling participants (1226 women and 862 men) and 1301 Chilean community-dwelling participants (855 women and 446 men). Sequential logistic regression analysis was used to examine the relationships between SRH and potential predictors in a hierarchical model.

Results: Overall, $35.5 \%$ and $52.1 \%$ of individuals in Chile and Brazil, respectively, reported good SRH. There was a gradient association between good SRH and chronic diseases in both countries. Chilean men without chronic disease or with one had a higher chance of good SRH, compared to two or more diseases. For Brazilian men, no or one chronic disease was associated with good SRH. For women, the set of independent predictors for good SRH included no chronic diseases or one chronic disease, and no activities of daily living limitation. For men, the set also included instrumental activities limitation. For Brazilian adults of both genders, depression demonstrated the strongest independent association with good SRH.

Conclusions: We conclude that when examining gender differences in predictors of SRH, the similarities are greater than the differences between Brazilian and Chilean older adults. In both countries, physical health was the most important predictor of SRH. In addition, absence of depression was the strongest predictor of good health in older Brazilian adults.
\end{abstract}

Keywords: Self-rated health, Older adults, Gender, Latin American

\section{Background}

In recent decades, Latin American countries have experienced a rapid process of demographic and epidemiological transitions leading to a rapidly ageing population [1]. In the last half century, the percentage of persons aged 60 years and over rose from $6 \%$ to $8 \%$. In 2025 , it is expected to swell to 56 million, bringing the total older population to 96 million. However, this trend is homogeneous neither at the international nor the national level [2]. In Chile, $11.5 \%$ of the population is older than 60 years and is expected to reach about $20.1 \%$ by 2025 [3]. In 2010-2015, the life expectancy at birth, currently the

\footnotetext{
* Correspondence: campos.acv@gmail.com

${ }^{1}$ School of Dentistry, Universidade Federal de Minas Gerais, Presidente Antônio Carlos 6627, Belo Horizonte, Minas Gerais 31270-901, Brazil Full list of author information is available at the end of the article
}

highest in Latin America, is estimated at 76.1 years for men and 82.2 years for women [4]. In Brazil, there are approximately 25 million people aged 60 or older $(10.8 \%$ of the population) [5]. Projections for 2030, estimate a life expectancy in Brazil around 77.4 years. In 2050 almost $30 \%$ of the Brazilian population will be 60 years and older, placing Brazil as one of the countries with the largest absolute number of older people worldwide [6].

However, ageing in both countries is occurring even in the context of health inequalities, with high rates of poverty, relative low coverage and quality of health and pension systems [7-10]. Among countries with available data, Brazil is among the countries with the worst income distribution in Latin America (Gini coefficient = 0.547), but Chile was no exception to this situation (Gini
C Biomed Central (c) 2015 Campos et al.; licensee BioMed Central. This is an Open Access article distributed under the terms of the Creative Commons Attribution License (http://creativecommons.org/licenses/by/4.0), which permits unrestricted use, distribution, and reproduction in any medium, provided the original work is properly credited. The Creative Commons Public Domain Dedication waiver (http://creativecommons.org/publicdomain/zero/1.0/) applies to the data made available in this article, unless otherwise stated. 
coefficient $=0.521)$ [11]. In addition, Brazilian illiteracy is high (24\%) [5] compared to the Chile (12\%) [1].

The characterisation of the health conditions of older people requires detailed information about different aspects of life, such as demographic and socioeconomic factors, chronic diseases and functional capacity $[8,12]$. In addition to acting as a social gradient, the unfavourable economic and social circumstances also affect health over the life span, making the health of older people even more susceptible to social determination by the accumulation of exposures to risk factors [13].

Self-rated health (SRH) is a multidimensional construct, which includes physical, psychological, functional and social variables [14] and has been used in population surveys in several countries [15-17]. The World Health Organization (WHO) recommended this indictor to verify health in population-based studies including older individuals.

In general, SRH among older adults is positive. The multicentre SABE study (Salud, Bienestar y Envejecimiento) [18] was conducted in the following seven cities of Latin America and the Caribbean: Buenos Aires, Argentina; Bridgetown, Barbados; Havana, Cuba; Montevideo, Uruguay; Santiago, Chile; Mexico City, DF, Mexico and São Paulo, Brazil. In São Paulo, 44.2\% of women and $48.4 \%$ of men reported good/very good SRH. In Santiago, this prevalence was lower for both sexes (33.9\% and $44.2 \%$, respectively). In other SABE countries, good SRH ranged from $27.9 \%$ among men in Mexico to 69.0\% among men in Uruguay [18].

The determinants of SRH have been widely investigated to explain social differences [19] and gender differences in health $[20,21]$. This study aimed to investigate the gender differences in predictors of SRH among Brazilian and Chilean older people.

\section{Methods \\ Participants}

This study concerns the analysis of the baseline cohort AGEQOL study and the Chilean sample of SABE.

"Aging, Gender and Quality of Life (AGEQOL)" is a cohort study in Sete Lagoas, Brazil with a representative sample of 2052 community-dwelling participants (1226 women and 862 men), aged 60 and older. Data collection was conducted between January and July 2012. The sampling process was conducted in two stages: in the first, census tracts were selected and in the second, households within each sector were selected. In each household, all residents aged 60 years or more of both genders, regardless of your marital status or kinship were interviewed. All persons $60+$ years in the selected households were informed of the study and were asked to sign an informed consent form that had been previously approved by the Ethical Committee of the Federal
University of Minas Gerais. The interviews lasted 40 to 60 minutes. At the end of the interviews, each subject in the city received guidance regarding health care and activity options as well as the personal contact information of the researcher responsible for the questionnaire [22].

The Chilean sample cohort SABE includes a representative sample of 1301 participants (855 women and 446 men) aged 60 and older living in private households in Santiago, Chile (January 2000 to January 2011). The primary sampling unit (PSU) was a conglomerate of independent households within a given geographical area. The PSU were divided in turn into secondary sampling units (SSU), each consisting of a smaller number of independent households. These USM joined in turn by tertiary sampling units (UTM), consisting of selected households that were interviewed all persons aged 60 or older. An informed consent to participate in this study should be obtained from all participants. The protocol for this study has been approved by the Institutional Review Board at Institute of Nutrition and Food Technology (INTA), University of Chile [18].

\section{Variables}

Most variables were dichotomised to enhance the interpretability of the logistic regression coefficients. For ordinal variables, dummy variables were created with the "worst" category used as the referent [23].

The outcome variable, SRH, was assessed with the question: 'How would you describe your health in the last 30 days?', with the response options "very good", "good", "fair", "poor" and "very poor" which were dichotomised as poor (fair/poor/very poor) versus good (very good/good).

The socioeconomic and demographic information included age ( $<75$ years, $\geq 75$ years), gender (male, female) marital status (married, separate, widower, single), retirement (yes, no), tertiles of income, years of education $(0$, $1-4,5-7, \geq 8$ ), and living arrangements (living with spouse, mixed arrangements, living alone). Current smoking, drinking, physical activity and social participation were measured as dichotomous variables (yes, no). The affordability of healthcare was measured by public and others.

Chronic diseases (hypertension, diabetes, cardiovascular diseases, musculoskeletal and respiratory diseases) is one of the indicators used to compare health among older people in different countries because it is an easy to obtain indicator that reflects multiple aspects of health that are difficult to capture using other methods in representative samples of the population [24]. The number of diseases was measured in three categories: 0 , $1, \geq 2$.

Functional capacity was evaluated from the participants' responses to six basic activities of daily living 
(ADL) - eating, dressing and undressing, grooming, walking, getting in and out of bed, bathing and continence [25] and seven instrumental activities (IADL) using the telephone, travel, shopping, meal preparation, housework, taking medicine and management of finances [26]. The participants were classified as restricted if they had one or more ADL limitation(s) or IADL limitation(s) [8]. To evaluate the cognitive status of older people, we used the Mini - Mental State Examination validated in Brazil [27] and in Chile [28], with a cut-off set at $21 / 22$ points [29]. Depressive symptoms were measured by the Geriatric Depression Scale short version (GDS-15) previously validated in Brazil [30] and in Chile [31] with a cut-off of $5 / 6$ points.

\section{Statistical analyses}

SPSS software (SPSS Institute, Chicago, USA) version 19.0 was used for the analysis including Chi-Square tests and logistic regression. Sequential logistic regression analysis was used to examine the relationships between SRH with potential predictors. The present study used the model from Demirchyan et al. [21] adapted to investigate the factors associated with good perceived health among older people. The independent variables were grouped into conceptually coherent hierarchical blocks: physical health, social structure, behavioural/attitudinal, psychosocial (Figure 1). In model 1, we examined the association between SRH and chronic diseases, ADL limitation, IADL limitation and cognitive impairment (physical health). In addition, we adjusted for variables of social structure (age, sex, marital status, education,

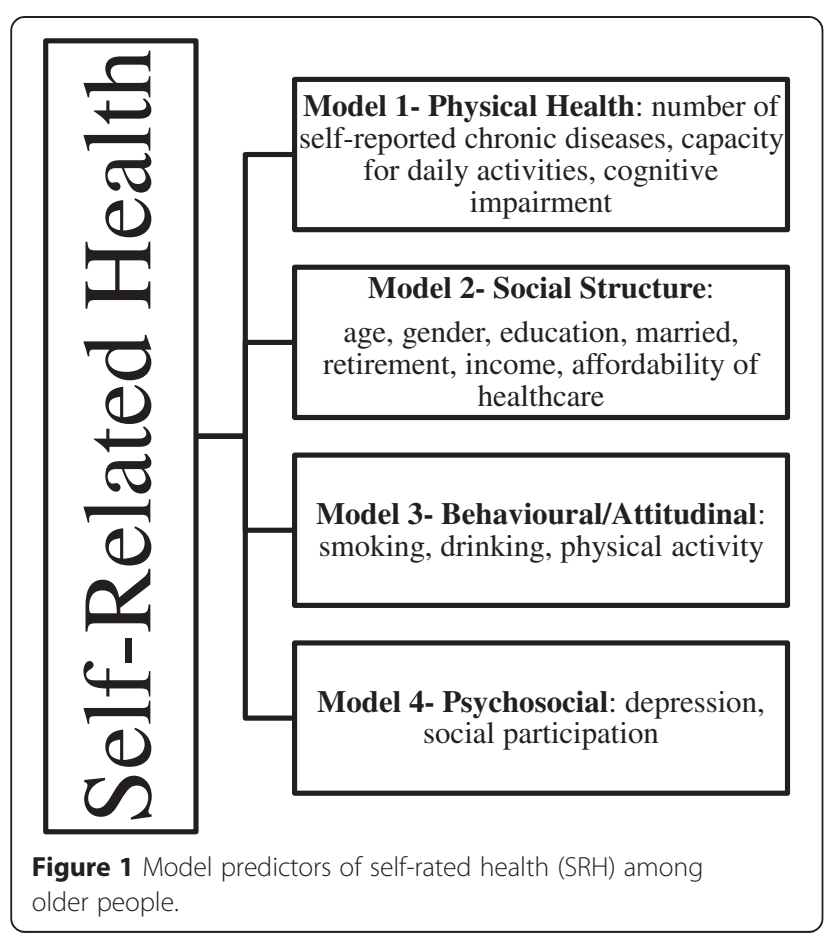

income, retired, household arrangement and healthcare) in Model 2. Smoking, drinking and physical activity comprised the third level of adjustment in Model 3 (behavioural/attitudinal). Finally, we adjusted for psychosocial level (depression and social participation) in Model 4. Considering that the sets of determinants of SRH could vary among women and men [20], we performed regression stratified by gender with the variables that remained in the final model.

Odds ratios (OR) and 95\% confidence intervals (CI95\%) for good SRH were calculated, and $\mathrm{p}$ values less than 0.05 (two-tailed test) were considered statistically significant. In addition, homogeneity and multicollinearity tests showed that all assumptions of the model were satisfied.

\section{Results}

\section{Descriptive characteristics}

In Brazilian sample the mean age was $70.9 \pm 8.1$ years (71.3 \pm 8.3 for women and $70.7 \pm 7.8$ for men). The mean age of the Chilean sample at the start of follow-up was $72 \pm 8.2$ years $(73.0 \pm 8.5$ women and $71.0 \pm 7.5$ for men $)$.

Table 1 provides descriptive statistics by gender for both countries. Most men $(70.8 \%$ for Brazilian and $72.2 \%$ for Chilean) and women $(68.7 \%$ for Brazilian and $62.7 \%$ for Chilean) were aged $60-74$ years old. Gender differences in ages only emerged among Chilean older people. Taking into account the three groups of living arrangements established in this study, there were $2.5 \%$ more women living alone in Brazil (16.6\%) and Chile (14.2\%) compared to men. For Brazilian older adults, we observed gender differences in living arrangements $(\mathrm{p}<0.001)$.

The percentage of illiteracy among Brazilian older people was higher than among Chilean older people, $28.2 \%$ and $17.1 \%$ respectively. These percentages were higher among Chilean women (19.7\%) and Brazilian men (29.1\%). There were gender differences in years of education among Chilean older adults $(p=0.002)$. In Brazil, only $10.4 \%$ of men and $8.6 \%$ of women reported over 4 years of study. By contrast, for Chilean older people, those percentages were $62.9 \%$ and $53.7 \%$, respectively (Table 1 ).

There were statistically significant gender differences for both countries related to marital status, income, retirement, current smoking and drinking. The great majority of men in the sample were married $(74.5 \%$ for Brazilian and $52.5 \%$ for Chilean). Most Brazilian older people had low monthly income (66.1\%), even higher among females (71.5\%) compared to males (58.1\%). The income of Chilean older people was more homogeneous (Table 1).

Only $15.9 \%$ and $10.5 \%$ of the Brazilian and Chilean older people did not have chronic disease, respectively. However, the percentage of women (59.6\% for Brazilian 
Table 1 Descriptive characteristics by gender

\begin{tabular}{|c|c|c|c|c|c|c|}
\hline \multirow[t]{2}{*}{ Variables } & \multicolumn{3}{|l|}{ Chile } & \multicolumn{3}{|l|}{ Brazil } \\
\hline & $\begin{array}{l}\text { Total } \\
(\mathrm{N}=1301)\end{array}$ & $\begin{array}{l}\text { Men } \\
(N=446)\end{array}$ & $\begin{array}{l}\text { Women } \\
(\mathrm{N}=855)\end{array}$ & $\begin{array}{l}\text { Total } \\
(\mathrm{N}=2052)\end{array}$ & $\begin{array}{l}\text { Men } \\
(\mathrm{N}=826)\end{array}$ & $\begin{array}{l}\text { Women } \\
(\mathrm{N}=1226)\end{array}$ \\
\hline \multicolumn{7}{|l|}{ Physical health } \\
\hline Chronic Diseases & n (\%) & n (\%) & n (\%) & n (\%) & n (\%) & n (\%) \\
\hline$\geq 2$ & $654(53)$ & $171(41.2)$ & $483(58.8)$ & 1099 (53.6) & $368(44.6)$ & $731(59.6)$ \\
\hline 1 & $452(36.6)$ & $182(44)$ & $270(32.9)$ & $626(30.5)$ & $274(33.2)$ & $352(28.7)$ \\
\hline 0 & $129(10.5)$ & $61(14.7)$ & $68(8.3)$ & $327(15.9)$ & $184(22.3)$ & $143(11.7)$ \\
\hline \multicolumn{7}{|l|}{ ADL Limitation } \\
\hline Yes & $251(19.3)$ & $45(10.1)$ & $206(24.1)$ & $160(7.8)$ & $98(8.0)$ & $62(7.5)$ \\
\hline No & $1044(80.3)$ & $398(89.2)$ & $646(75.6)$ & $1892(92.2)$ & $764(92.5)$ & $1128(92.0)$ \\
\hline \multicolumn{7}{|l|}{ IADL Limitation } \\
\hline Yes & $374(28.9)$ & $78(17.6)$ & $296(34.7)$ & $600(29.2)$ & $196(23.7)$ & $404(33.0)$ \\
\hline No & $922(71.1)$ & $365(82.4)$ & $557(65.3)$ & $1452(70.8)$ & $633(76.3)$ & $822(67.0)$ \\
\hline \multicolumn{7}{|c|}{ Cognitive Impairment } \\
\hline Yes & $118(9.1)$ & $30(6.7)$ & $88(10.3)$ & $264(12.9)$ & $98(11.9)$ & $166(13.5)$ \\
\hline No & $1183(90.9)$ & $416(93.3)$ & $767(89.7)$ & $1788(87.1)$ & $728(88.1)$ & $1060(86.5)$ \\
\hline \multicolumn{7}{|l|}{ Social structure } \\
\hline \multicolumn{7}{|l|}{ Age } \\
\hline$<75$ years old & $858(65.9)$ & $322(72.2)$ & $536(62.7)$ & $1427(69.5)$ & $585(70.8)$ & $842(68.7)$ \\
\hline$\geq 75$ years old & $443(34.1)$ & $124(27.8)$ & $319(37.3)$ & $579(28.2)$ & $241(29.2)$ & $339(27.7)$ \\
\hline \multicolumn{7}{|l|}{ Marital Status } \\
\hline Married & $451(34.7)$ & $234(52.5)$ & $217(25.4)$ & $1084(52.9)$ & $615(74.5)$ & $469(38.3)$ \\
\hline Separate & $249(19.1)$ & $86(19.3)$ & $163(19.1)$ & $154(7.5)$ & $59(7.1)$ & $95(7.8)$ \\
\hline Widower & $397(46.4)$ & $107(24)$ & $504(38.7)$ & $612(29.9)$ & $93(11.3)$ & $519(42.4)$ \\
\hline Single & $95(7.3)$ & $18(4)$ & $77(9)$ & $199(9.7)$ & $59(7.1)$ & $140(11.4)$ \\
\hline \multicolumn{7}{|l|}{ Years of Education } \\
\hline 0 & $222(17.1)$ & $54(12.1)$ & $168(19.7)$ & $579(28.2)$ & $240(29.1)$ & $339(27.7)$ \\
\hline $1-4$ & $339(26.1)$ & $111(24.9)$ & $228(26.7)$ & $1282(62.5)$ & $500(60.5)$ & $782(63.8)$ \\
\hline $5-7$ & $365(28.1)$ & $133(29.8)$ & $232(27.1)$ & $130(6.3)$ & $63(7.6)$ & $67(5.5)$ \\
\hline$>8$ & $375(28.8)$ & $148(33.1)$ & $227(26.6)$ & $61(3.0)$ & $23(2.8)$ & $38(3.1)$ \\
\hline \multicolumn{7}{|l|}{ Income } \\
\hline $1^{\circ}$ tertile & $361(30.7)$ & $91(21.5)$ & $270(35.9)$ & $1357(66.1)$ & $480(58.1)$ & $877(71.5)$ \\
\hline $2^{\circ}$ tertile & $384(32.7)$ & 125(29.6) & $259(34.4)$ & $505(24.6)$ & $246(29.8)$ & $259(21.1)$ \\
\hline $3^{\circ}$ tertile & $430(36.6)$ & 207 (48.9) & 223(29.7) & $190(9.3)$ & $100(12.1)$ & $90(7.3)$ \\
\hline \multicolumn{7}{|l|}{ Retired } \\
\hline Yes & $397(33.5)$ & $160(36)$ & $237(31.9)$ & $1518(74.0)$ & $699(84.6)$ & $819(66.8)$ \\
\hline No & $790(66.5)$ & $285(64)$ & $505(68.1)$ & $534(26.0)$ & $27(15.4)$ & $407(33.2)$ \\
\hline \multicolumn{7}{|c|}{ Household Arrangement } \\
\hline Living with spouse & $284(21.8)$ & $94(21.1)$ & $149(16.3)$ & $1065(53.0)$ & $616(75.5)$ & 449 (37.6) \\
\hline Mixed arrangements & $851(65.4)$ & $307(68.8)$ & $544(63.6)$ & $668(33.2)$ & $121(14.8)$ & $547(45.8)$ \\
\hline Living alone & $166(12.8)$ & $45(10.1)$ & $121(14.2)$ & $277(13.8)$ & $79(9.7)$ & $198(16.6)$ \\
\hline \multicolumn{7}{|l|}{ Healthcare } \\
\hline Public & $1089(83.7)$ & $359(80.5)$ & $730(85.4)$ & $1153(56.2)$ & $477(57.7)$ & $676(55.1)$ \\
\hline Others & $63(4.8)$ & $25(5.6)$ & $38(4.4)$ & $899(43.8)$ & $349(42.3)$ & $550(44.9)$ \\
\hline
\end{tabular}


Table 1 Descriptive characteristics by gender (Continued)

\begin{tabular}{|c|c|c|c|c|c|c|}
\hline Beha & & & & & & \\
\hline Curre & & & & & & \\
\hline Yes & $158(12.2)$ & 78 (17.6) & $80(6.7)$ & $225(11.0)$ & $143(17.3)$ & $82(6.7)$ \\
\hline No & 1139 (87.8) & 365 (82.4) & 774 (90.6) & $1827(89.0)$ & $683(82.7)$ & 1144 (93.3) \\
\hline Drinl & & & & & & \\
\hline Yes & $92(7.1)$ & 65 (14.6) & $27(3.2)$ & 383 (18.7) & $171(17.4)$ & 212 (19.8) \\
\hline No & $1206(92.9)$ & 379 (85.4) & 827 (96.8) & 1669 (81.3) & $569(68.9)$ & 1100 (89.7) \\
\hline Phys & & & & & & \\
\hline Yes & $270(20.8)$ & $121(27.2)$ & 149 (17.5) & 545 (26.6) & $216(26.2)$ & $329(26.8)$ \\
\hline No & $1028(79.2)$ & $324(72.8)$ & 704 (82.5) & 1507 (73.4) & $610(73.8)$ & 897 (73.2) \\
\hline Psycl & & & & & & \\
\hline Sym & & & & & & \\
\hline Yes & 854 (26.3) & $92(22.7)$ & $213(28.3)$ & $619(30.2)$ & 197 (23.8) & $422(34.4)$ \\
\hline No & 854 (73.7) & 314 (77.3) & $540(71.7)$ & 1433 (69.8) & 785 (95.0) & 804 (65.6) \\
\hline Socia & & & & & & \\
\hline Yes & 408 (31.5) & $108(24.3)$ & $300(35.2)$ & $157(7.7)$ & $41(5.0)$ & $116(9.5)$ \\
\hline No & 889 (68.5) & $336(75.7)$ & $553(64.8)$ & 1895 (92.3) & 900 (91.6) & $1110(90.5)$ \\
\hline
\end{tabular}

ADL: activities of daily living.

IADL: instrumental activities of daily living.

and $58.8 \%$ for Chilean) with more than two diseases was statistically higher $(\mathrm{p}<0.001)$ compared to men $(44.6 \%$ for Brazilian and $41.2 \%$ for Chilean). The prevalence of cognitive impairment was $12.9 \%$ among Brazilian adults and $9.1 \%$ among Chilean, with significant gender differences (Table 1).

In relation to depression, there was a $30.2 \%$ prevalence of depressive symptoms among Brazilian older people and a statistically significant $(\mathrm{p}<0.001)$ difference between the genders $(23.8 \%$ for men and $34.4 \%$ for women). For Chilean people, we observed a significantly higher percentage among females $(28.3 \%)$ compared to males (22.7\%). We observed more Chilean women with ADL limitations $(\mathrm{p}<0.001)$. The prevalence of IADL limitations was similar between the two countries, $29.2 \%$ for Brazil and $28.9 \%$ for Chile. There were significant differences $(\mathrm{p}<0.001)$ between men $(23.7 \%$ for Brazilian and $10.1 \%$ for Chilean) and women (33.0\% for Brazilian and $24.1 \%$ for Chilean) (Table 1).

\section{Results of bivariate analysis}

Overall, $35.5 \%$ and $52.1 \%$ of individuals reported good $\mathrm{SRH}$, in Chile and Brazil, respectively. The distribution of SRH according to physical health, social structure, behavioural/attitudinal and psychosocial factors is shown in Table 2. Poor SRH responses were significantly more frequent among women (64.7\% for Brazilian and 69.2\% for Chilean) than men.

In Table 2, statistically significant differences for SRH were found in both countries related to chronic diseases,
ADL limitation, IADL limitation, cognitive impairment, sex, education, income, healthcare and physical activity. The frequency of good SRH was 2.3 times higher among non-retired Chilean older people compared to retired ( $\mathrm{p}<$ 0.001). In Brazilian older adults, household arrangement and marital status were associated with good SRH. Brazilian older adults without depression had better perception of health compared to those with depression $(\mathrm{p}<0.001)$.

\section{Hierarchical model of predictors of SRH}

We estimated the OR of good SRH in regression models. Brazilian and Chilean adults without symptoms of depression had $3.3(\mathrm{OR}=3.3, \mathrm{CI} 95 \%=2.5-4.0)$ and $1.4(\mathrm{OR}=1.4$, CI95\% $=1.0-2.0)$ higher odds of good SRH, compared with symptoms of depression (Additional files 1 and 2).

Good SRH was significantly predicted by no ADL limitation $(\mathrm{OR}=2.1 ; \mathrm{CI} 95 \%=1.0-4.3)$, no IADL limitation $(\mathrm{OR}=1.8 ; \quad \mathrm{CI} 95 \%=1.1-3.0), \quad<75$ years old $(\mathrm{OR}=1.6$; CI95\% $=1.1-2.3)$, 8 years of education $(\mathrm{OR}=1.7$; CI95\% =1.0-2.8), and healthcare $(\mathrm{OR}=2.1 ; \mathrm{CI} 95 \%=1.1-4.2)$. Older people with one or two chronic disease(s) had 3.6 and 2.2 higher chance of good SRH, compared to no chronic disease (Additional file 1).

There was an inverse gradient for association between good SRH and chronic diseases in all models. When controlling for potential confounds, the associations were attenuated but remained statistically significant. One chronic disease $(\mathrm{OR}=2.4, \mathrm{CI} 95 \%=1.9-3.0)$ and, in particular, no chronic disease $(\mathrm{OR}=4.1, \mathrm{CI} 95 \%=3.0-5.5)$, were associated with an elevated chance of good SRH (Additional file 2). 
Table 2 Descriptive characteristics of self-related health among Brazilians and Chileans older adults

\begin{tabular}{|c|c|c|c|c|c|c|}
\hline \multirow[t]{2}{*}{ Variables } & \multicolumn{3}{|c|}{ Self-related health Chile $(N=1300)$} & \multicolumn{3}{|c|}{ Self-related health Brazil $(\mathrm{N}=2052)$} \\
\hline & $\begin{array}{l}\text { Poor } \\
(\mathrm{N}=839)\end{array}$ & $\begin{array}{l}\text { Good } \\
(N=461)\end{array}$ & $\mathrm{p}$ & $\begin{array}{l}\text { Poor } \\
(\mathrm{N}=982)\end{array}$ & $\begin{array}{l}\text { Good } \\
(N=1070)\end{array}$ & $\mathrm{p}$ \\
\hline \multicolumn{7}{|l|}{ Physical health } \\
\hline Chronic Diseases & n (\%) & n (\%) & & n (\%) & n (\%) & \\
\hline$\geq 2$ & $488(61.3)$ & $165(37.7)$ & $<0.001$ & $670(68.2)$ & $429(40.1)$ & $<0.001$ \\
\hline 1 & $247(31)$ & $205(46.8)$ & & $231(23.5)$ & $395(36.9)$ & \\
\hline 0 & $61(7.7)$ & $68(15.5)$ & & $81(8.2)$ & $246(23.0)$ & \\
\hline \multicolumn{7}{|l|}{ ADL Limitation } \\
\hline Yes & $74(11.5)$ & $33(7.2)$ & $<0.001$ & $113(11.5)$ & $47(4.4)$ & $<0.001$ \\
\hline No & $618(88.5)$ & $426(92.8)$ & & $869(88.5)$ & $1023(95.6)$ & \\
\hline \multicolumn{7}{|l|}{ IADL Limitation } \\
\hline Yes & $605(36.4)$ & $68(14.9)$ & $<0.001$ & $349(35.5)$ & $251(23.5)$ & $<0.001$ \\
\hline No & $533(63.6)$ & $389(85.1)$ & & $633(64.5)$ & $819(76.5)$ & \\
\hline \multicolumn{7}{|l|}{ Cognitive Impairment } \\
\hline Yes & $91(10.9)$ & $26(5.6)$ & 0.002 & $149(15.2)$ & $115(10.7)$ & 0.003 \\
\hline No & $748(89.1)$ & $435(94.4)$ & & $833(84.8)$ & $955(89.3)$ & \\
\hline \multicolumn{7}{|l|}{ Social structure } \\
\hline \multicolumn{7}{|l|}{ Age } \\
\hline$<75$ years old & $545(65.0)$ & $313(67.9)$ & 0.285 & $669(68.1)$ & $758(70.8)$ & 0.195 \\
\hline$\geq 75$ years old & $294(35.0)$ & $148(32.1)$ & & $313(31.9)$ & $312(29.2)$ & \\
\hline \multicolumn{7}{|l|}{ Sex } \\
\hline Masculine & $258(30.8)$ & $188(40.8)$ & $<0.001$ & 347 (35.3) & $479(44.8)$ & $<0.001$ \\
\hline Feminine & $581(69.2)$ & $273(59.2)$ & & $635(64.7)$ & $591(55.2)$ & \\
\hline \multicolumn{7}{|l|}{ Marital Status } \\
\hline Married & $282(33.6)$ & 169 (36.8) & 0.439 & $487(49.7)$ & $597(55.8)$ & 0.004 \\
\hline Separate & $156(18.6)$ & $92(20)$ & & $66(6.7)$ & $88(8.2)$ & \\
\hline Widower & $336(40.1)$ & 168 (36.6) & & $327(33.4)$ & $285(26.7)$ & \\
\hline Single & $65(7.8)$ & $30(6.5)$ & & $100(10.2)$ & $99(9.3)$ & \\
\hline \multicolumn{7}{|l|}{ Years of Education } \\
\hline 0 & 164 (19.6) & $58(12.6)$ & $<0.001$ & $295(30.0)$ & $284(26.5)$ & $<0.001$ \\
\hline $1-4$ & $231(27.5)$ & $107(23.2)$ & & $626(63.7)$ & $656(61.3)$ & \\
\hline $5-7$ & $243(29)$ & $122(26.5)$ & & $44(4.5)$ & $86(8.0)$ & \\
\hline$>8$ & $201(24)$ & $174(37.7)$ & & $17(1.7)$ & $44(4.1)$ & \\
\hline \multicolumn{7}{|l|}{ Income } \\
\hline $1^{\circ}$ tertile & $265(35)$ & $96(23)$ & $<0.001$ & $697(71.0)$ & $660(61.7)$ & $<0.001$ \\
\hline $2^{\circ}$ tertile & $250(33)$ & $133(32)$ & & $219(22.3)$ & $286(26.7)$ & \\
\hline $3^{\circ}$ tertile & $243(32)$ & $187(45)$ & & $66(6.7)$ & $124(11.6)$ & \\
\hline \multicolumn{7}{|l|}{ Retired } \\
\hline Yes & $269(35.4)$ & $128(30.0)$ & $<0.001$ & $722(73.5)$ & $796(74.4)$ & 0.687 \\
\hline No & $490(64.6)$ & $299(70.0)$ & & $260(26.5)$ & $274(25.6)$ & \\
\hline \multicolumn{7}{|c|}{ Household Arrangement } \\
\hline Living with spouse & $184(21.9)$ & $99(21.5)$ & 0.654 & $479(49.5)$ & $586(56.2)$ & 0.010 \\
\hline Mixed arrangements & $543(64.7)$ & $308(66.8)$ & & 345 (35.6) & $323(31.0)$ & \\
\hline
\end{tabular}


Table 2 Descriptive characteristics of self-related health among Brazilians and Chileans older adults (Continued)

\begin{tabular}{|c|c|c|c|c|c|c|}
\hline Living alone & $112(13.4)$ & $54(11.7)$ & & $144(14.9)$ & $133(12.8)$ & \\
\hline \multicolumn{7}{|l|}{ Healthcare } \\
\hline Public & 719 (59.4) & 369 (53.3) & $<0.001$ & $583(59.4)$ & $570(53.3)$ & 0.003 \\
\hline Others & $28(3.3)$ & $35(7.6)$ & & 399 (40.6) & $500(46.7)$ & \\
\hline \multicolumn{7}{|c|}{ Behavioural/attitudinal } \\
\hline \multicolumn{7}{|c|}{ Currently Smoking } \\
\hline Yes & $94(11.2)$ & $64(13.9)$ & 0.153 & $116(11.8)$ & $109(10.2)$ & 0.258 \\
\hline No & $743(88.8)$ & $395(86.1)$ & & $866(88.2)$ & 961 (89.8) & \\
\hline \multicolumn{7}{|l|}{ Drinking } \\
\hline Yes & $46(5.5)$ & $46(10.0)$ & 0.002 & $171(17.4)$ & $212(19.8)$ & 0.174 \\
\hline No & $793(94.5)$ & $412(90.0)$ & & 811 (82.6) & $858(80.2)$ & \\
\hline \multicolumn{7}{|c|}{ Physical Activity } \\
\hline Yes & $150(17.9)$ & $120(26.1)$ & $<0.001$ & $208(21.2)$ & $337(31.5)$ & $<0.001$ \\
\hline No & $688(82.1)$ & 339 (73.9) & & 774 (78.8) & $733(68.5)$ & \\
\hline \multicolumn{7}{|c|}{ Psychosocial } \\
\hline \multicolumn{7}{|c|}{ Symptoms of Depression } \\
\hline Yes & $207(28.2)$ & $98(23.1)$ & 0.055 & $430(43.8)$ & $189(17.7)$ & $<0.001$ \\
\hline No & $527(71.8)$ & $327(76.9)$ & & $552(56.2)$ & 881 (82.3) & \\
\hline \multicolumn{7}{|c|}{ Social Participation } \\
\hline Yes & $248(29.7)$ & $160(34.8)$ & 0.058 & $82(8.4)$ & $75(7.0)$ & 0.280 \\
\hline No & $588(66.2)$ & $300(65.2)$ & & $900(91.6)$ & 995 (93.0) & \\
\hline
\end{tabular}

ADL: activities of daily living.

IADL: instrumental activities of daily living.

No ADL limitation, no IADL limitation, 5-7 years of education and no smoking were also strongly associated with good SRH, while all other variables were comparable. By contrast, no physical activity was a risk factor for poorer health perception (Additional file 2). In model 1 , Brazilian adults without cognitive impairment had 1.4 higher probability of having good SRH than with cognitive impairment $(\mathrm{p}=0.031)$. However, this association did not remain in the final model (Additional file 2).

\section{Gender analysis}

Table 3 presents the results of significant associations between perceived health and predictors for females and males separately. There was a gradient association between good SRH and chronic diseases in both countries. Chilean men without chronic disease or with one had 3.3 and 2.9 higher chance of good SRH, compared to those with two or more diseases. For Brazilian men, no chronic disease $(\mathrm{OR}=4.4, \mathrm{CI} 95 \%=2.9-6.8)$ and one $(\mathrm{OR}=2.6$, C195\% $=1.8$-3.6) were associated with good SRH (Table 3).

For women, the set of independent predictors of good SRH included no chronic diseases $(\mathrm{OR}=3.9$ for Brazilian and $\mathrm{OR}=4.4$ for Chilean) or one chronic ( $\mathrm{OR}=2.2$ for Brazilian and $\mathrm{OR}=2.1$ for Chilean) disease, and no ADL limitation ( $\mathrm{OR}=2.5$ for both) (Table 3$)$.
For men, the set also included no IADL limitation. The influence of functional limitation in health perception was 1.5 higher among Chileans adults $(\mathrm{OR}=3.0, \mathrm{CI} 95 \%=1.0$ 9.0) than Brazilian adults ( $\mathrm{OR}=1.5, \mathrm{CI} 95 \%=1.1-2.2)$ (Table 3). Brazilian men who did not exercise had $70 \%$ higher probability of having poor SRH than men with physical activity (Table 3). For Brazilian adults of both genders, depression demonstrated the strongest independent association with good SRH. Men and women without depression had almost three times more chance of having good SRH $(\mathrm{OR}=2.8$ and $\mathrm{OR}=3.1$, respectively), compared to those with depression (Table 3 ).

\section{Discussion}

This is one of the few studies from South American countries to study the effects of socioeconomic status and health on SRH among older adults when attempting to understand health inequalities between men and women.

Good SRH was 1.5 times higher among Brazilian older adults $(52.1 \%)$ compared to Chileans (35.5\%). Data from the SABE study indicated poorer health among older people of both sexes in the cities of São Paulo, Santiago and Mexico - countries with high levels of income inequalities [32]. A systematic review of SRH in Brazilian 
Table 3 Final model predicting good self-related health in older adults separated by gender

\begin{tabular}{|c|c|c|c|c|c|c|c|c|}
\hline & \multicolumn{4}{|c|}{ Chile $^{a}$} & \multicolumn{4}{|c|}{ Brazil $^{b}$} \\
\hline & \multicolumn{2}{|l|}{ Male } & \multicolumn{2}{|c|}{ Female } & \multicolumn{2}{|l|}{ Male } & \multicolumn{2}{|c|}{ Female } \\
\hline & OR & $95 \% \mathrm{Cl}$ & OR & $95 \% \mathrm{Cl}$ & OR & $95 \% \mathrm{Cl}$ & OR & $95 \% \mathrm{Cl}$ \\
\hline \multicolumn{9}{|c|}{ Chronic Diseases } \\
\hline 0 & $3.3^{* *}$ & $1.9-5.7$ & $4.4^{* *}$ & $2.2-8.9$ & $4.4^{* *}$ & $2.9-6.8$ & $3.9^{* *}$ & $2.6-6.0$ \\
\hline 1 & $2.9^{*}$ & $1.3-6.0$ & $2.1^{* *}$ & $1.4-3.2$ & $2.6^{* *}$ & $1.8-3.6$ & $2.2^{* *}$ & $1.7-2.9$ \\
\hline$\geq 2$ & 1.0 & & 1.0 & & 1.0 & & 1.0 & \\
\hline \multicolumn{9}{|c|}{ ADL Limitation } \\
\hline No & 1.0 & $0.2-5.4$ & $2.5^{*}$ & $1.1-5.5$ & 1.1 & $0.6-2.0$ & $2.5^{*}$ & $1.4-5.0$ \\
\hline Yes & 1.0 & & 1.0 & & 1.0 & & 1.0 & \\
\hline \multicolumn{9}{|c|}{ IADL Limitation } \\
\hline No & $3.0^{*}$ & $1.0-9.0$ & 1.2 & $0.7-2.4$ & $1.5^{*}$ & $1.1-2.2$ & 1.0 & $0.8-1.4$ \\
\hline Yes & 1.0 & & 1.0 & & 1.0 & & 1.0 & \\
\hline \multicolumn{9}{|c|}{ Physical Activity } \\
\hline No & 0.7 & $0.4-1.2$ & 0.8 & $0.5-1.2$ & $0.7^{*}$ & $0.5-1.0$ & 0.8 & $0.6-1.1$ \\
\hline Yes & 1.0 & & 1.0 & & 1.0 & & 1.0 & \\
\hline \multicolumn{9}{|c|}{ Depression } \\
\hline No & 1.0 & $0.6-1.7$ & 1.5 & $0.9-2.3$ & $2.8^{* *}$ & $2.0-4.1$ & $3.1^{* *}$ & $2.4-4.1$ \\
\hline Yes & 1.0 & & 1.0 & & 1.0 & & 1.0 & \\
\hline
\end{tabular}

${ }^{a}$ Controlling for age, education, income, retirement, healthcare, alcohol.

${ }^{\mathrm{b}}$ Controlling for education, smoking.

${ }^{*} \mathrm{p}<0.05$.

${ }^{* *} \mathrm{p}<0.001$.

older people showed a prevalence of negative SRH between $12.6 \%$ and $51.9 \%$ [33].

These differences between the studies may have two possible explanations. The first is the absence of standardised questions and response options regarding SRH. In a study conducted in Armenia, the categories of response were "excellent", "very good", "good", "fair" and "poor" which were dichotomised as fair/poor versus good [21]. In other studies, the authors chose to analyse very poor, poor and fair together versus very good/good $[19,32]$. These categorisations may overestimate the prevalence of negative or positive SRH. Secondly, comparisons are difficult given the different definitions used, as no reference values have been defined in the literature as acceptable for SRH among older people [33].

Women had worse health perception compared to older men in Brazil and Chile. These results were in agreement with previous studies conducted in Latin American and the Caribbean [24,32,34], and in others countries $[21,35]$. On other hand, this study contradicted other studies demonstrating better perception of health among women $[19,36]$.

SRH has been shown to be a reliable method for measuring gender differences in health status [37] and a good predictor of mortality [38,39] among older people. However, in our study, multivariate analyses showed no independent association between gender and SRH in Brazil or in Chile.

The influence of gender in relation to older people's health and SRH was confirmed in other studies [15,35] and further investigation is needed. This study provided evidence in the model separated by gender that SRH differed between men and women and varied between Brazil and Chile.

There was a consistent inverse relationship between chronic disease and SRH among Brazilians and Chileans older people. More interesting is that these differences remained when other variables were included in the model and when the results were separated by gender. Brazilian men and Chilean women seemed to report the highest SRH in the absence of chronic health, even after adjusting for confounds. Data from São Paulo in SABE indicated that in the absence of chronic diseases, or in the presence of two or more chronic diseases, older women self-assessed their health relatively better than older men [19].

This difference regarding the effect of gender on SRH countries may be related to differences in the sex ratio or in both samples. In Chile, there were 1.9 women for every man while the ratio for the sample in Brazil was 1.5. In addition, these data referred to both baseline studies. Therefore, it is not possible to determine whether these 
gender differences are cultural or whether there is a temporal relationship between SRH and gender and other variables.

In both countries, we observed the strongest association between ADL and IADL limitation with SRH, controlling for chronic diseases and cognitive impairment. In model 4, these associations were attenuated but remained statistically significant. Good SRH can be considered a protective factor against functional limitations $[19,24,40]$.

Among women, no ADL limitation was statistically associated with good SRH. A majority of women who reported good health had no ADL limitation in Brazil (95.6\%) and Chile (92.8\%). Among men, no IADL limitation was statistically associated with good SRH. The chance among Chilean older people $(\mathrm{OR}=1.5 ; \mathrm{CI} 195 \%=$ 1.1-2.2) was twice as high than among Brazilians $(\mathrm{OR}=$ 3.0; $\mathrm{CI} 95 \%=1.0-9.0$ ).

The prevalence of functional limitations in older people was high, particularly among women $[8,12,41]$. While in Chile the prevalence of ADL limitation was higher among women, in Brazil there was an inverse relationship between the genders. Regarding IADL, the prevalence of limitation was higher among women compared to men in both countries.

Overall prevalence of depression was higher in Brazilian older people (30.2\%) that Chilean older people (26.3\%), but associations between SRH and depression were only found in Brazil . The chance of older people without depression reporting better health was slightly higher among women. This could be explained by the high prevalence of depression in women (34.4\%), and, in particular, among women with poor SRH (72.0\%). A study conducted in Spain showed the strongest relation to poor SRH with depressive symptoms $(\mathrm{OR}=5.0)$, with important differences between genders (women, $\mathrm{OR}=$ 4.7 and men, $\mathrm{OR}=5.2$ ) [14].

Depression among women is a complex phenomenon that deserves to be investigated in relation to other health conditions and age [42]. We suggest that future studies can incorporate variables such as menopause, anxiety and clinical measures of women's health between each age group (60-64 years, 65-69 years, 7074 years and $>75$ years). We believe that considering gender differences among the ageing may contribute to the improvement of SRH and, ultimately, the welfare of this group.

\section{Limitations}

SRH is among the most frequently assessed health perceptions in epidemiological research. However it is necessary to discuss some options for standardising questions and answers in order to follow and compare results to guide decisions about the best health policies for Latin America and the world [33]. In a study comparing different measures of SRH with respect to differences in age and sex groups, the authors concluded that noncomparative measures were more appropriate in longitudinal studies and that measures without specified response options might be less suitable for a study with older people [43].

In this study, we did not find any association between socioeconomic status, gender and SRH. The socioeconomic factors discussed in this study were limited to income and education. Furthermore, categorised income tertiles were measured in different currencies which were converted to U.S. dollars to compare the results. It is necessary to include other contextual (consumer goods, purchasing power) and cultural (local habits and customs) characteristics. Future research should also focus on the health effects of gendered differences in domestic and paid work, and on home and family roles as well as the interaction among gender, household crowding, and health [44].

\section{Conclusions}

In conclusion, when examining gender differences in predictors of SRH, the similarities are greater than the differences between Brazilian and Chilean older people. In both countries, physical health was the most important predictor of SRH. In addition, the absence of depression was the strongest predictor of good health in Brazilian older people.

\section{Additional files}

Additional file 1: Logistic Regression results for physical health, social structure, behavioural/attitudinal and psychosocial variables predicting good self-related health in Chilean older adults.

Additional file 2: Logistic regression results for physical health, social structure, behavioural/attitudinal and psychosocial variables predicting good self-related health in Brazilian older adults.

Competing interests

The authors declare that they have no competing interests.

\section{Authors' contributions}

ACVC conducted Brazilian data analyses and drafted the manuscript. AMDV and EFF contributed to the conception and design of the study. LL conducted Chilean data analyses and participated in the interpretation of the data. HS and CA participated in the interpretation and discussion of the data and made critical revisions to the manuscript. All authors read and approved the final manuscript.

\section{Acknowledgements}

The AGEQOL study was supported by the National Council for Scientific and Technological Development - CNPq [481672/2011-7, 305032/2012-7,

141307/2011-0]; the Coordination of Improvement of Higher Education CAPES [process 8314/13-6]; Pró-Reitoria de Pesquisa da Universidade Federal de Minas Gerais - PRPq/UFMG; and Fundação de Amparo à Pesquisa de Minas Gerais - FAPEMIG. The SABE study was supported by the National Fund for Science and Technology [project Fondecyt 1080589]. 


\section{Author details}

${ }^{1}$ School of Dentistry, Universidade Federal de Minas Gerais, Presidente Antônio Carlos 6627, Belo Horizonte, Minas Gerais 31270-901, Brazil. unidad Nutrición, Salud Pública y Envejecimiento Saludable, INTA, Universidad de Chile, El Líbano 5524 Macul, Santiago 138-11, Chile. ${ }^{3}$ Department of Community and Preventive Dentistry, School of Dentistry, Universidade Federal de Minas Gerais, Presidente Antônio Carlos 6627, Belo Horizonte, Minas Gerais 31270-901, Brazil.

\section{Received: 6 November 2014 Accepted: 19 March 2015}

\section{Published online: 11 April 2015}

\section{References}

1. Albala C, Vio F. Epidemiological transition in Latin America: the case of Chile. Public Health. 1995;109:431-42.

2. Comisión Económica para América Latina y el Caribe/Centro Latinoamericano y Caribeño de Demografia. América Latina y el Caribe: estimaciones y proyecciones de población. 1950-2050. Santiago de Chile: Comisión Económica para América Latina y el Caribe, Naciones Unidas; 2004

3. Chile. Proyecciones y estimaciones de población, Total país período información 1950-2050. Chile [internet]. 2005. Instituto Nacional de Estadísticas INE, CELADE. Serie de la publicación (CEPAL): OI N²08. [cited 2013 Oct 30]. Available from: http://www.ine.cl/canales/chile_estadistico/ demografia_y_vitales/proyecciones/Informes/MicrosoftWordInforP_T.pdf.

4. Departamento de Estadísticas e Información de Salud. Esperanza de vida a nacer (en años) por periodo y sexo. Chile, 1950-2025. Santiago: Ministerio de Salud; 2011.

5. Instituto Brasileiro de Geografia e Estatística. Search national household sample. Brazil [Internet]. 2010 [cited 2011 May 02]. Available from: http:// www.ibge.gov.br/home/estatistica/populacao/censo2010/ default_resultados_universo.shtm

6. Beltrão Kl, Camarano AA, Kanso S. Brazilian population dynamics at the turn of the century XX. Rio de Janeiro: IPEA; 2004

7. Paskulin LMG, Aires M, Gonçales AV, Kottwitz CCB, Morais EP, Brondani MA Aging, diversity, and health: the Brazilian and the Canadian context. Acta Paul Enferm. 2011;24(6):851-6.

8. Fuentes-García A, Sánchez H, Lera L, Cea X, Albala C. Desigualdades socioeconómicas en el proceso de discapacidad en una cohorte de adultos mayores de Santiago de Chile. Gac Sanit. 2013;27:226-32.

9. Albala C, Sánchez H, Lera L, Angel B, Cea X. Efecto sobre la salud de las desigualdades socioeconómicas en el adulto mayor: Resultados basales del estudio expectativa de vida saludable y discapacidad relacionada con la obesidad (Alexandros). Rev Med Chil. 2011;139:1276-85.

10. Sánchez H, Albala C. Desigualdades en salud: mortalidad del adulto en comunas del Gran Santiago. Rev Méd Chile. 2004:132:453-60.

11. The World Bank. Indicators. Gini Index. [Internet] [cited 2012 Apr 4]. Available from: http://data.worldbank.org/indicator/ SI.POV.GINI?display=default

12. Drumond Andrade FC, Guevara PE, Lebrao ML, de Oliveira Duarte YA Santos JL. Gender differences in life expectancy and disability-free life expectancy among older adults in Sao Paulo, Brazil. Womens Health Issues. 2011:21:64-70.

13. Geib LTC. Determinantes sociais da saúde do idoso. Ciênc saúde coletiva. 2012;17(1):123-33

14. Millán-Calenti JC, Sánchez A, Lorenzo T, Maseda A. Depressive symptoms and other factors associated with poor self-rated health in the elderly: gender differences. Geriatr Gerontol Int. 2012;12(2):198-206.

15. French DJ, Browning C, Kendig H, Luszcz MA, Saito Y, Sargent-Cox K, et al. A simple measure with complex determinants: investigation of the correlates of self-rated health in older men and women from three continents. BMC Public Health. 2012;12:649.

16. Kunst $A E$, Geurts JJM, van den Berg J. International variation in socioeconomic inequalities in self reported health. J Epidemiol Community Health. 1995:49(2):117-23.

17. Kunst AE, Bos V, Lahelma E, Bartley M, Lissau I, Regidor E, et al. Trends in socioeconomic inequalities in self-assessed health in 10 European countries. Int J Epidemiol. 2005;34(2):295-305.

18. Albala C, Lebrão ML, León Díaz EM, Ham-Chande R, Hennis AJ, Palloni A, et al. Encuesta Salud, Bienestar y Envejecimiento (SABE): metodología de la encuesta y perfil de la población estudiada. Rev Panam Salud Publica. 2005;17:307-22

19. Alves $L S$, Rodrigues $R N$. Determinants of self-rated health among elderly persons in São Paulo, Brazil. Rev Panam Salud Publica. 2005;17:333-41.

20. Borrell C, Muntaner C, Benach J, Artazcoz L. Social class and self-reported health status among men and women: what is the role of work organization, household material standards and household labor? Soc Sci Med. 2004:58(10):1869-87.

21. Demirchyan A, Petrosyan V, Thompson ME. Gender differences in predictors of self-rated health in Armenia: a population-based study of an economy in transition. Int J Equity Health [Internet]. 2012;11:67. Available from: http:// www.equityhealthj.com/content/11/1/67.

22. Campos ACV, Vargas AMD, Ferreira EF. Oral health satisfaction among Brazilian elderly: a gender study using a hierarchical model. Cad Saúde Pública. 2014:30(4):757-73.

23. Hosmer DW, Lemeshow S. Applied logistic regression. 2nd ed. New York: A Wiley-Interscience Publication; 2000.

24. Wong R, Peláez M, Palloni A. Autoinforme de salud general en adultos mayores de América Latina y el Caribe: su utilidad como indicador. Rev Panam Salud Publica. 2005;17(5-6):323-32

25. Katz S, Akpom CA. A measure of primary sociobiological functions. Int J Health Serv. 1976;6:493-509.

26. Lawton MP, Brody EM. Assessment of older people: Self maintaining and instrumental activities of daily living. Gerontologist. 1969;9:179-86.

27. Brucki SMD, Nitrini R, Caramelli P, Bertolucci PHF, Okamoto $\mid H$. Sugestões para o uso do mini-exame do estado mental no Brasil. Arq Neuropsiquiatr. 2003;61:77-81.

28. Albala C, Icaza G, Vio F, García C, Marín PP, Quiroga P, et al. A short test to evaluate cognitive impairment based on Folstein's MMSE. Gerontology. 2001:47(S1):S183.

29. Quiroga P, Albala C, Klaasen. Validación de un test de tamizaje para el diagnóstico de demencia asociada a edad, en Chile. Rev Med Chile. 2004;132:467-78

30. Almeida OP, Almeida AS. Confiabilidade da versão brasileira da escala de depressão em geriatria (GDS) versão reduzida. Arq Neuropsiquiatr. 1999:57:421-6.

31. Hoyl T, Valenzuela E, Marín PP. Depression in the aged: preliminary evaluation of the effectiveness, as an screening instrument, of the 5-item version of the Geriatric Depression Scale. Rev Med Chile. 2000;128:1199-204.

32. Zunzunegui MV, Alvarado BE, Béland F, Vissandjee B. Explaining health differences between men and women in later life: a cross-city comparison in Latin America and the Caribbean. Soc Sci Med. 2009:68(2):235-42.

33. Pagotto $V$, Bachion MM, Silveira EA. Autoavaliação da saúde por idosos brasileiros: revisão sistemática da literatura. Rev Panam Salud Publica. 2013:33(4):302-10

34. Olivares-Tirado P, Leyton G, Salazar E. Personality factors and self-perceived health in Chilean elderly population. Health [Internet]. 2013;|.5(12A):86-96. Available from: http://dx.doi.org/10.4236/health.2013.512A012.

35. Molarius A, Granstrom F, Feldman I, Blomqvist MK, Pettersson H, Elo S. Can financial insecurity and condescending treatment explain the higher prevalence of poor self-rated health in women than in men? A populationbased cross-sectional study in Sweden. Int J Equity in Health [Internet]. 2012;11(50). Available from: http://www.ncbi.nlm.nih.gov/pmc/articles/ PMC3441386/pdf/1475-9276-11-50.pdf.

36. Sun W, Watanabe M, Tanimoto Y, Shibutani T, Kono R, Saito M, et al. Factors associated with good self-rated health of non-disabled elderly living alone in Japan: a cross-sectional study. BMC Public Health. 2007;7:297.

37. Singh L, Arokiasamy P, Singh PK, Rai RK. Determinants of gender differences in self-rated health among older population: evidence From India. SAGE Open [Internet]. 2013;1-12. Available from: http://sgo.sagepub.com/content/ 3/2/2158244013487914

38. Bath PA. Differences between older men and women in the self-rated health-mortality relationship. Gerontologist. 2003;43(3):387-95. discussion 372-5.

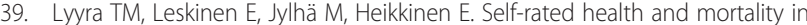
older men and women: a time-dependent covariate analysis. Arch Gerontol Geriatr. 2009;48(1):14-8.

40. Gama EV, Damián JE, Pérez de Molino J, López MR, López Pérez M, Gavira Iglesias FJ. Association of individual activities of daily living with self-rated health in older people. Age Ageing. 2000;29:267-70.

41. Alexandre Tda S, Corona LP, Nunes DP, Santos JL, Duarte YA, Lebrão ML. Gender differences in incidence and determinants of disability in activities 
of daily living among elderly individuals: SABE study. Arch Gerontol Geriatr. 2012;55:431-7.

42. Faravelli C, Alessandra Scarpato M, Castellini G, Lo Sauro C. Gender differences in depression and anxiety: the role of age. Psychiatry Res. 2013;210:1301-3.

43. Eriksson I, Undén AL, Elofsson S. Self-rated health. Comparisons between three different measures. Results from a population study. Int J Epidemiol. 2001;30(2):326-33.

44. Dunn JR, Walker JD, Graham J, Weiss CB. Gender differences in the relationship between housing, socioeconomic status, and self-reported health status. Rev Environ Health. 2004;19(3-4):177-95.

Submit your next manuscript to BioMed Central and take full advantage of:

- Convenient online submission

- Thorough peer review

- No space constraints or color figure charges

- Immediate publication on acceptance

- Inclusion in PubMed, CAS, Scopus and Google Scholar

- Research which is freely available for redistribution 